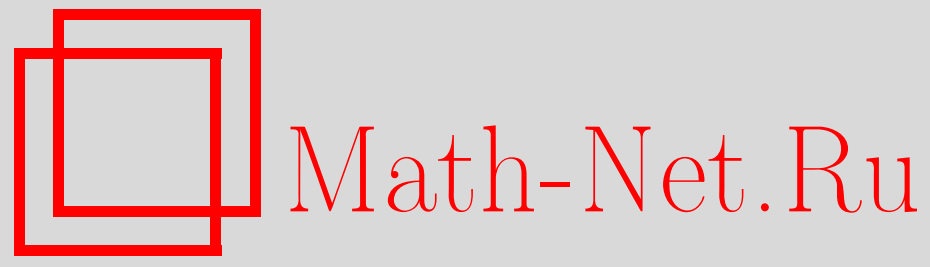

Р. С. Исмагилов, Слабые $\mathrm{KKC}$ и $\mathrm{KAC}$ и индуктивные пределы семейств групп и алгебр, Функи. анализ и его прил., 2000, том 34, выпуск 2, 75-78

DOI: https://doi.org/10.4213/faa300

Использование Общероссийского математического портала MathNet.Ru подразумевает, что вы прочитали и согласны с пользовательским соглашением

http://www . mathnet.ru/rus/agreement

Параметры загрузки:

IP : 54.198 .64 .247

26 апреля 2023 г., 12:56:46

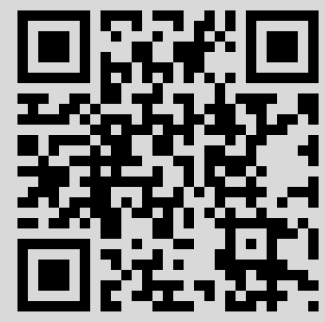


ЗАмечАниЕ. Точный вектор $d$, соответствующий нетривиально оснащенному однопараметрическому множеству $\mathbf{P}$, является особълм тогда и только тогда, когда множество $\mathbf{P}$ (рассматриваемое с точностью до антиизоморфизма) совпадает с одним из множеств $M_{12}, M_{13}, L_{14}, L_{15}$ и $d=\left(d_{0} ; d_{a}, d_{b}, d_{x}, d_{y}\right)=$ $(2 k+1 ; k, k, 1,1)$ при $\mathbf{P}=M_{12}, d=\left(d_{0} ; d_{a}, d_{b}, d_{p}, d_{q}\right)=(2 k+2 ; k, k, 1,1)$ при $\mathbf{P}=M_{13}, d=\left(d_{0} ; d_{a}, d_{u}, d_{v}, d_{x}, d_{y}\right)=(2 k+1 ; k, k, k, 1,1)$ при $\mathbf{P}=L_{14}, d=$ $\left(d_{0} ; d_{a}, d_{u}, d_{v}, d_{p}, d_{q}\right)=(4 k+2 ; 2 k, 2 k, 2 k, 1,1)$ при $\mathbf{P}=L_{15}(k \geqslant 1)$. При этом $f(d)=2$ в случае $\mathbf{P}=M_{12}, L_{14}$ и $f(d)=4$ в случае $\mathbf{P}=M_{13}, L_{15}$ (об особых векторах обычных однопараметрических множеств см., например, [11]).

В ходе работы получен также полный список неразложимых представлений всех перечисленных множеств (и им антиизоморфных) в матричной форме.

Доказательство основано на разработке и применении алгоритмов дифференцирования оснащенных множеств (развивающих алгоритмы, описанные в $[1,11])$, использовании результатов работы [12] и непосредственных вычислениях.

Авторы благодарны рецензенту за полезные замечания.

\title{
ЛИтеРАтУРА
}

1. Назарова Л. А., Ройтер А. В. Зап. научн. сем. ЛОМИ, 28, 5-31 (1972). 2. Назарова Л. A., Ройтер А. В. Препринт 73.9 ИМ АН УССР (1973). 3. Gabriel P., Roiter A. $V$. Representations of finite-dimensional algebras. Algebra VIII, Encycl. Math. Sci., vol. 73, Springer-Verlag, 1992. 4. Dlab V., Ringel C. M. Indecomposable representations of graphs and algebras. Mem. Amer. Math. Soc., 6, No. 173 (1976). 5. Poümep A. B. Труды МИАН, 148, 201-210 (1978). 6. Клейнер М. М. Зап. научн. сем. ЛОМИ, 28, 32-41 (1972). 7. Клейнер М. М. Зап. научн. сем. ЛОМИ, 28, 42-59 (1972). 8. Отрашевская В. В. Укр. матем. ж., 28, № 3, 334-341 (1976). 9. Отрашевская В. В. В сб.: Матричные задачи, Киев, 1977, с. 144-149. 10. Klemp B., Simson D. J. Algebra, 134, No. 2, 390-468 (1990). 11. Завадский А. Г. Изв. АН СССР, сер. матем., 55, № 5, 10071048 (1991). 12. Dlab V., Ringel C. M. Linear Algebra Appl., 17, 107-124 (1977).

Киевский национальный университет

строительства и архитектуры

Поступило в редакцию 30 декабря 1997 г.

УДК $515.16+517.9$

\section{Слабые ККС и КАС и индуктивные пределы семейств групп и алгебр*}

\author{
(c) 2000. Р. С. ИСМАГИЛов
}

1. Еще о канонических коммутационных и антикоммутационных соотношениях (кратко $\mathrm{KKC}$ и $\mathrm{KAC}$ ). Пусть $V$ - вещественное линейное пространство и $\tau$ - невырожденная кососимметрическая форма на $V$. Тогда $\mathrm{KKC} \mathrm{-} \mathrm{это} \mathrm{семейство} \mathrm{унитарных} \mathrm{операторов}\{U(x), x \in V\}$ (действующих в некотором гильбертовом пространстве), таких, что

$$
U(x+y)=\exp (i \tau(x, y)) U(x) U(y) \quad(x, y \in V)
$$

* Исследование поддержано РФФИ, грант № 96-15-96249. 
и отображение $s \mapsto U(s x) U(s y), s \in \mathbb{R}$, слабо непрерывно.

Разумеется, из (1) вытекает равенство

$$
U(x+y)=U(x) U(y) \quad \text { при } x \perp y
$$

(здесь $x \perp y$ означает, что $\tau(x, y)=0$ ). Верно ли обратное? Точнее, пусть $\{U(x)$, $x \in V\}$ - неприводимое семейство унитарных операторов, удовлетворяющее условию (2) и упомянутому условию непрерывности; в этом случае скажем, что даны слабые КKС. Выполняется ли тогда соотношение (1) (разумеется, с возможной заменой формы $\tau$ на $\lambda \tau, \lambda \in \mathbb{R})$ ?

Аналогично, определим слабые $\mathrm{KKC}$ в инфинитезимальной форме как семейство симметрических операторов $\{A(x), x \in V\}$, действующих в плотном линейном подмножестве гильбертова пространства, таких, что

$$
\text { если } x \perp y \text {, то }[A(x), A(y)]=0, A(\alpha x+\beta y)=\alpha A(x)+\beta A(y)(\alpha, \beta \in \mathbb{R}) .
$$

(Подчеркнем, что линейность отображения $x \mapsto A(x)$ не требуется!) Наложим еще условие операторной неприводимости: любой оператор, представимый в виде многочлена степени $\leqslant 2$ от операторов $\{A(x)\}$ и перестановочный с ними, скалярен. Вытекают ли отсюда линейность отображения $x \mapsto A(x), x \in V$, и обычные коммутационные соотношения $[A(x), A(y)]=i \lambda \tau(x, y)$ для некоторого $\lambda \in \mathbb{R}$ ?

Обратимся к KАС. Пусть $W$ - комплексное линейное пространство с инволюцией $x \mapsto \bar{x}$ и невырожденной симметрической формой $\theta$, причем $\theta(x, \bar{x})>0$ при $x \neq 0$ (иногда мы пишем $x \perp y$ вместо $\theta(x, y)=0$ ). Определим слабые КАС как семейство ограниченных операторов $\{C(x), x \in W, x \perp x\}$, таких, что $(C(x))^{*}=C(\bar{x})$ и

$$
\begin{gathered}
\text { если } x \perp y, x \perp x, y \perp y, \\
\text { то }\{C(x), C(y)\}=0, C(\alpha x+\beta y)=\alpha C(x)+\beta C(y)(\alpha, \beta \in \mathbb{C}) .
\end{gathered}
$$

Пусть семейство $\{C(x)\}$ неприводимо. Можно ли продолжить семейство $\{C(x)\}$ до $\mathbb{C}$-линейного отображения $x \mapsto C^{0}(x)$ в пространство ограниченных линейных операторов, такого, что выполняются обычные $\mathrm{KAC}\left\{C^{0}(x), C^{0}(y)\right\}=$ $\lambda \theta(x, y)$ для всех $x \in W, y \in W$ (и некоторого $\lambda \geqslant 0$, не зависящего от $x, y)$ ?

Мы увидим, что во всех трех случаях ответ на вопрос утвердителен. Этот результат получится из «вычисления» некоторых индуктивных пределов семейств групп и алгебр. Затем мы кратко остановимся на примерах индуктивных пределов, не связанных с $\mathrm{KKC}$ и $\mathrm{KAC}$.

2. Индуктивные пределы. Мы воспроизведем определение из [1]. Пусть $\left\{G_{\alpha}\right\}$ - набор групп и для любых $\alpha, \beta$ дан набор (возможно, пустой) гомоморфизмов $\varphi_{\alpha \beta}: G_{\alpha} \rightarrow G_{\beta}$. Индуктивный предел семейства $\left\{G_{\alpha}\right\}$ состоит из группы $\Gamma^{0}$ и набора гомоморфизмов $f_{\alpha}^{0}: G_{\alpha} \rightarrow \Gamma^{0}$, удовлетворяющих следующим условиям: 1) $f_{\alpha}^{0}=f_{\beta}^{0} \circ \varphi_{\alpha \beta}$, если только определен $\left.\varphi_{\alpha \beta} ; 2\right)$ множество $\bigcup_{\alpha} f_{\alpha}^{0}\left(G_{\alpha}\right)$ порождает группу $\left.\Gamma^{0} ; 3\right)$ для любых $\Gamma$ и $\left\{f_{\alpha}\right\}$, удовлетворяющих двум предыдущим условиям, существует такой гомоморфизм $p: \Gamma^{0} \rightarrow \Gamma$, что $f_{\alpha}=p \circ f_{\alpha}^{0}$ для всех $\alpha$.

Отметим важный частный случай: $\left\{G_{\alpha}\right\}$ - семейство подгрупп данной группы $G$, замкнутое относительно пересечений и порождающее группу $G$, и $\varphi_{\alpha \beta}: G_{\alpha} \rightarrow G_{\beta}$ - включение $\left(\varphi_{\alpha \beta}\right.$ определено только при $\left.G_{\alpha} \subset G_{\beta}\right)$. В этом 
случае набор гомоморфизмов, упомянутый выше, отождествляется с отображением $f: \bigcup_{\alpha} G_{\alpha} \rightarrow \Gamma$, ограничение которого на каждую группу $G_{\alpha}$ есть гомоморфизм.

Для алгебр (в частности, алгебр Ли) индуктивный предел определяется аналогично.

Перейдем к примерам.

3. Группа Гейзенберга как индуктивный предел абелевых групп; алгебра Гейзенберга. Пусть $V$ - линейное пространство над полем $K$, char $K \neq 2$, а $\tau$ - невырожденная кососимметрическая форма на $V$. Группа Гейзенберга $H$ - это множество $V \times K$ с операцией $\left(v_{1}, k_{1}\right) \cdot\left(v_{2}, k_{2}\right)=$ $\left(v_{1}+v_{2}, k_{1}+k_{2}+\tau\left(v_{1}, v_{2}\right)\right)$, а алгебра Гейзенберга - то же множество со скобкой Ли $\left[\left(v_{1}, k_{1}\right),\left(v_{2}, k_{2}\right)\right]=\left(0, \tau\left(v_{1}, v_{2}\right)\right)$. Через $\operatorname{Is}(V, \tau)$ обозначим совокупность всех изотропных линейных подпространств (т. е. подпространств, на которых форма $\tau$ тождественно равна нулю). В приведенной ниже теореме 1 мы рассматриваем Is $(V, \tau)$ как семейство абелевых (операция - сложение векторов), а в теореме 2 - как семейство абелевых алгебр Ли. Предполагается, что $\operatorname{dim} V \geqslant 4$.

Теорема 1. Индуктивный предел семейства групn $\operatorname{Is}(V, \tau)$ - это группа Гейзенберга $H$ ( с гомоморфизмами $x \mapsto(x, 0), x \in L, L \in \operatorname{Is}(V, \tau))$.

Теорема 2. Индуктивный предел семейства алгебр Ли $\operatorname{Is}(V, \tau)$ - это алгебра Гейзенберга $H$ ( с теми же гомоморфизмами $x \mapsto(x, 0), x \in L, L \in$ $\operatorname{Is}(V, \tau))$.

4. Индуктивный предел грассмановых подалгебр алгебры Клиффорда. Пусть $W$ - линейное пространство над алгебраически замкнутым полем $K$, char $K \neq 2, \theta$ - невырожденная симметрическая форма на $W$ и $C(W)$ - соответствующая клиффордова алгебра. Любое изотропное подпространство $X$ порождает грассманову подалгебру $G(X)$. Требуется найти индуктивный предел семейства $\{G(X)\}$ (гомоморфизм $G(X) \rightarrow G(Y)$ определен при $X \subset Y)$.

Для этого обозначим через $C(W, T)$ ассоциативную алгебру с единицей, порожденную пространством $W$, переменной $T$ и соотношениями $x y+y x=$ $\theta(x, y) T, x T=T x$. Для любого изотропного пространства $X \subset W$ тождественное вложение $X \rightarrow C(W, T)$ продолжается до гомоморфизма алгебр. Предполагается, что $\operatorname{dim} W \geqslant 4$.

Теорема 3. Индуктивный предел семейства грассмановых алгебр $\{G(X)\}$ $(X$ изотропно) есть алгебра $C(W, T)$ ( с указанными гомоморфизмами $G(X) \rightarrow$ $C(W, T))$.

5. Равносильность слабых и обычных ККС и КАС. Вернемся к соотношениям (2) из разд. 1. Они задают отображение из $V$ в группу унитарных операторов, которое является гомоморфизмом на любом изотропном подпространстве из $V$, понимаемом как абелева группа. По определению индуктивного предела и теореме 1 существует такое унитарное представление группы Гейзенберга $x \mapsto U^{0}(x, 0)$, что $U^{0}(x, 0)=U(x), x \in V$. Из условий неприводимости и непрерывности следует, что $U^{0}(0, k)=\exp (i \lambda k), k \in \mathbb{R}$, для некоторого $\lambda \in \mathbb{R}$. Отсюда получаем обычные $\mathrm{KKC}$ для $\{U(x)\}$.

Аналогично доказывается равносильность слабых и обычных соотношений в остальных случаях. 
6. Другие примеры вычисления индуктивных пределов. Пусть $\mathfrak{g}$ компактная простая алгебра Ли, $\mathrm{Ab}(\mathfrak{g})$ - семейство ее абелевых подалгебр (гомоморфизмы - это включения). Требуется найти индуктивный предел семейства $\mathrm{Ab}(\mathfrak{g})$. Чтобы избежать патологических явлений, здесь следует внести в определение индуктивного предела естественные топологические ограничения. В случае $\mathfrak{g}=\mathrm{su}(n), n \geqslant 3$, искомый индуктивный предел - это алгебра Ли многочленов $x_{1} T+\cdots+x_{r} T^{r}, x_{i} \in \mathfrak{g}$, с вложениями $x \mapsto x T, x \in L, L \in \operatorname{Ab}(\mathfrak{g})$.

В работах $[2,3]$ рассмотрены индуктивные пределы групп диффеоморфизмов и алгебр Ли векторных полей.

\title{
ЛИТЕРАТУРА
}

1. Серр Ж. П. Математика (сб. переводов), 18, № 1, 3-51 (1974). 2. Исмагилов Р. С. Изв. АН СССР, сер. матем., 44, № 4, 831-867 (1980). 3. Исмагилов Р. С. Функц. анализ и его прил., 26, вып. 1, 66-68 (1992).

Московский государственный технический университет им. Н. Э. Баумана

Поступило в редакцию 30 апреля 1998 г.

\section{УДК 517.9}

\section{О задаче подобия для несамосопряженных операторов с абсолютно непрерывным спектром*}

\author{
(C) 2000. А. В. КИСЕЛЕВ, М. М. ФАДДЕЕВ
}

Оператор $L$, действующий в гильбертовом пространстве $H$, подобен самосопряженному, если существуют такие самосопряженный оператор $A$ и ограниченный ограниченно обратимый оператор $X$ в $H$, что $L=X^{-1} A X$. При условии вещественности спектра оператора $L$ для подобия оператора $L$ самосопряженному необходимо и достаточно $[1,2]$, чтобы

$$
\sup _{\varepsilon>0} \varepsilon \int\left\|(L-k-i \varepsilon)^{-1} u\right\|^{2} d k \leqslant C\|u\|^{2}
$$

и оценка (1) выполнялась также с заменой оператора $L$ на $L^{*}$. Данные оценки могут быть выписаны [3] в терминах функциональной модели оператора, однако их вид становится чрезвычайно громоздким. Мы можем привести их к значительно более простой форме, если на исследуемый оператор налагается дополнительное условие абсолютной непрерывности его спектра. В этом случае оказывается возможным привести указанные оценки к паре эквивалентных оценок уже для граничных значений резольвенты исследуемого оператора на вещественной оси, фактически реализовав в (1) предельный переход при $\varepsilon \rightarrow 0$, что, вообще говоря, затруднительно без предположения об абсолютной непрерывности спектра оператора.

Опишем вкратце функциональную модель исследуемого оператора в форме Б. С. Павлова (см. $[4,5,6])$. Рассмотрим наряду с оператором $L=A+i V \equiv$ $A+i \alpha J \alpha / 2$, где $A=A^{*}, \alpha=\alpha^{*} \geqslant 0$, оператор $V$ является $(A)$-ограниченным 